\title{
Phase shifting in an oblique incidence interferometer
}

\author{
Doris Boebel* \\ Robert Bosch GmbH \\ Postfach 1342 \\ 7410 Reutlingen \\ Federal Republic of Germany \\ Bernd Packro $\beta$ \\ Hans J. Tiziani, FELLOW SPIE \\ Institut für Technische Optik \\ Universität Stuttgart \\ Pfaffenwaldring 9 \\ 7000 Stuttgart 80 \\ Federal Republic of Germany
}

\begin{abstract}
The evaluation of the interference pattern of a prism interferometer by a phase-stepping technique is described. The required phase shift is achieved by moving the surface under test perpendicular to the hypotenuse of the prism. Limits of this phase-shifting technique are discussed. A calibration method of the phase shift between the interferograms for the adjustable sensitivity of the interferometer is shown. Finally measurements of technical surfaces are presented.
\end{abstract}

Subject terms: oblique incidence interferometers; fringe analysis; phase-stepping technique; variable fringe density; optically rough surfaces.

Optical Engineering 30(12), 1910-1914 (December 1991).

\section{CONTENTS}

1. Introduction

2. Experimental setup

3. Phase calculation and decompression of image deformation

4. Experimental results

5. Conclusion

6. References

\section{INTRODUCTION}

Twyman-Green and Fizeau interferometers are widely used for measurements of optical surfaces with high accuracy. The wave vector $\mathbf{k}$ in these interferometers is perpendicular to both the test and reference surfaces. One fringe in the resulting interference pattern represents a height of half a wavelength on the surface under test. These methods are, in general, too sensitive to measure technical surfaces. The surface slopes are often so high that the fringes cannot be resolved. Another disadvantage is the low specular reflection of optically rough surfaces at normal incidence.

To measure technical surfaces oblique incidence interferometers are used. The main advantage of the oblique incidence is a longer effective wavelength and thus a reduction of fringe density. According to the higher specular reflection at oblique incidence, interference patterns with good contrast are produced.

The oblique incidence interferometer described in this paper utilizes the hypotenuse of a right-angle prism as reference surface and beamsplitter. Abramson introduced the word "interferoscope" for this kind of interferometer with variable sensitivity. An advantage of this setup is the low image compression in the axis of incidence compared to other oblique incidence interfer-

*Author was with the Institut für Technische Optik, Universität Stuttgart, Pfaffenwaldring 9, 7000 Stuttgart 80, FRG, when this work was performed.

Paper 2954 received July 26, 1990; revised manuscript received March 14, 1991; accepted for publication April 6, 1991.

C 1991 Society of Photo-Optical Instrumentation Engineers. ometers. The distortion of the object is reduced by the refraction of the wavefront at the hypotenuse face of the prism. Further applications of Abramson's instrument are described in Refs. 2 through 5 . In the presented setup a piezo-driven sample mount was introduced as a phase-shifting device for digital calculation of the wavefront by a four interferogram algorithm.

\section{EXPERIMENTAL SETUP}

A schematic diagram of the interferometer is shown in Fig. 1. The light source is a He-Ne laser with a wavelength of $\lambda=632.8$ $\mathrm{nm}$. The plane wavefront after the beam expander (lens L1, L2) is reflected by the plane mirror M1 into the right-angle glass prism. The hypotenuse face acts as both the reference surface and the beamsplitter. Interference occurs between the wavefront reflected by the hypotenuse and the wavefront reflected by the surface under test. The interference pattern is imaged by lens $\mathrm{L} 3$ and a zoom lens onto a CCD camera and shown on a video

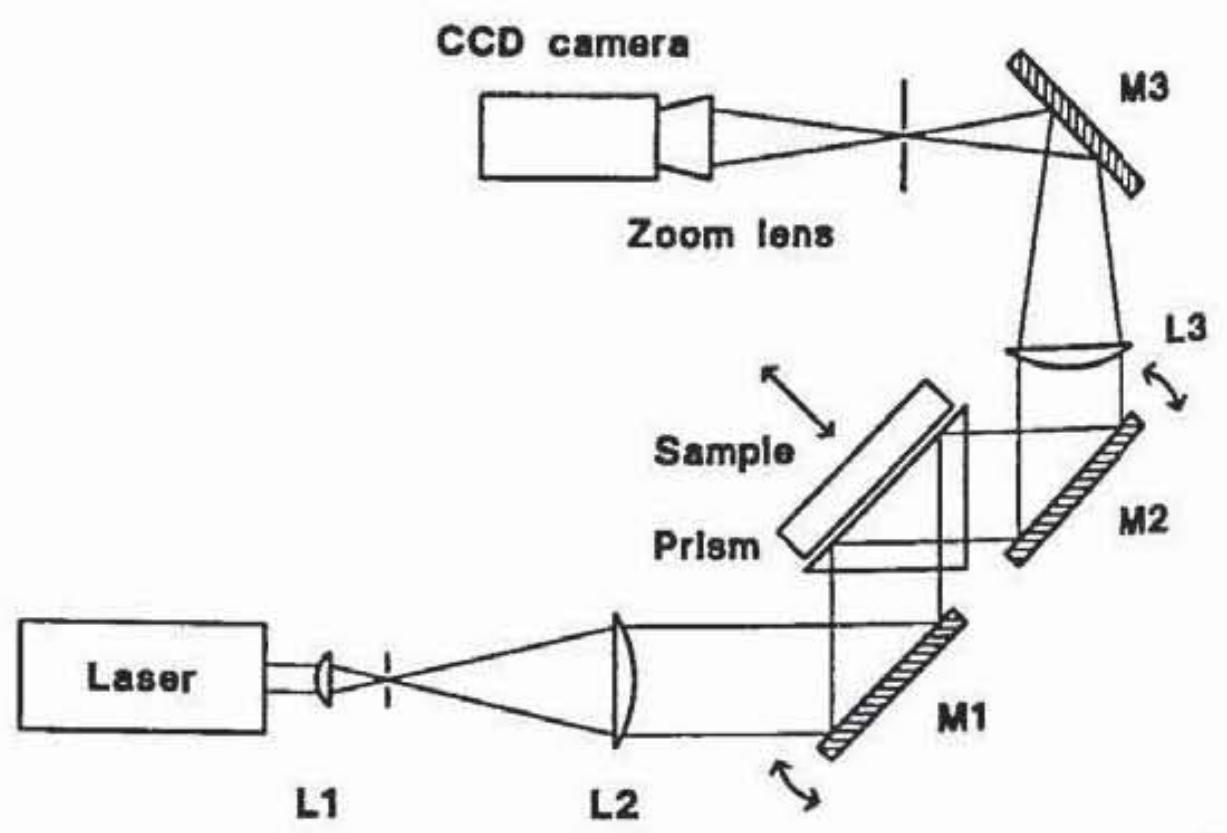

Fig. 1. Schematic diagram of the prism interferometer with continuous adjustment of the fringe separation and phase shift. 
monitor. A diaphragm is placed in the focal plane of L3 for image filtering.

Referring to Fig. 2, the optical path difference (OPD) between a reference and test beam is given by

$\mathrm{OPD}=A B C-n A D=2 h \sin \alpha$.

Thus the phase difference $\phi$ between the two beams is given by $\phi=\frac{2 \pi}{\lambda} 2 h \sin \alpha$.

The sensitivity of the oblique incidence interferometer is reduced by a factor of $\sin \alpha$ compared to the sensitivity of a normal incidence (e.g., Fizeau) interferometer. The effective wavelength $\lambda_{\text {eff }}=\lambda / \sin \alpha$ can be varied with the angle $\alpha$ on the surface under test. This interferometer is designed to measure with an effective wavelength $\lambda_{\text {eff }}$ from 1 to $10 \mu \mathrm{m}$. In order to obtain this wavelength range the angle $\alpha$ needs to be varied between 3.6 and $40 \mathrm{deg}$.

For this range a mirror holder was designed to select the sensitivity continuously. The angle of M1 relative to the incident plane wavefront determines the refraction angle $\beta^{\prime}$ behind the hypotenuse of the prism and thus the interferometric sensitivity. The mirror M2 is mechanically coupled to M1 in a way to minimize the shift of the imaged interference pattern when changing the sensitivity.

A short distance between the surface under test and the reference surface minimizes the sensitivity to environmental disturbances. A drawback is the occurrence of multiple beam interference due to the high reflectivity of the surfaces at oblique angles of incidence. For accurate calculation of the topography of a sample with a usual phase measurement algorithm, cosinusoidal interference fringes are required. This requirement is fulfilled for two beam interference patterns. A method to avoid multiple beam interferences and to generate cosinusoidal interference fringes in a prism interferometer is shown in Fig. 3. The maximum length of the measuring area is given by

$s=\frac{1}{2} c \frac{\cos \gamma^{\prime}}{\cos \beta}$,

with $c$ the length of the cathete of the right angle prism. The air gap is set to

$h=\frac{s}{2} \tan \alpha$.

Thus two beam interference fringes occur by the superposition of waves 1 and 2 reflected by the surface under test with the wavefront between beams 2 and 3 reflected by the hypotenuse of the prism.

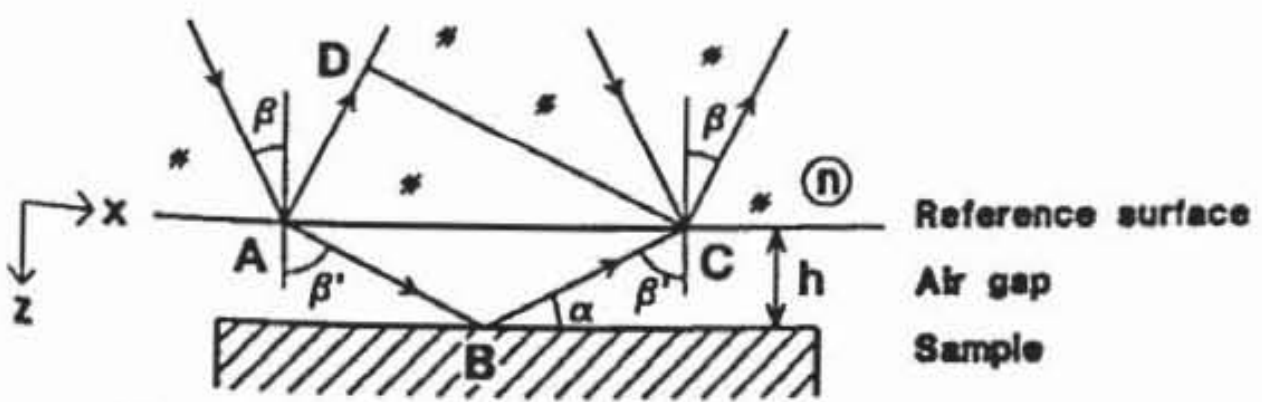

Fig. 2. Optical paths of the reference and test beam.

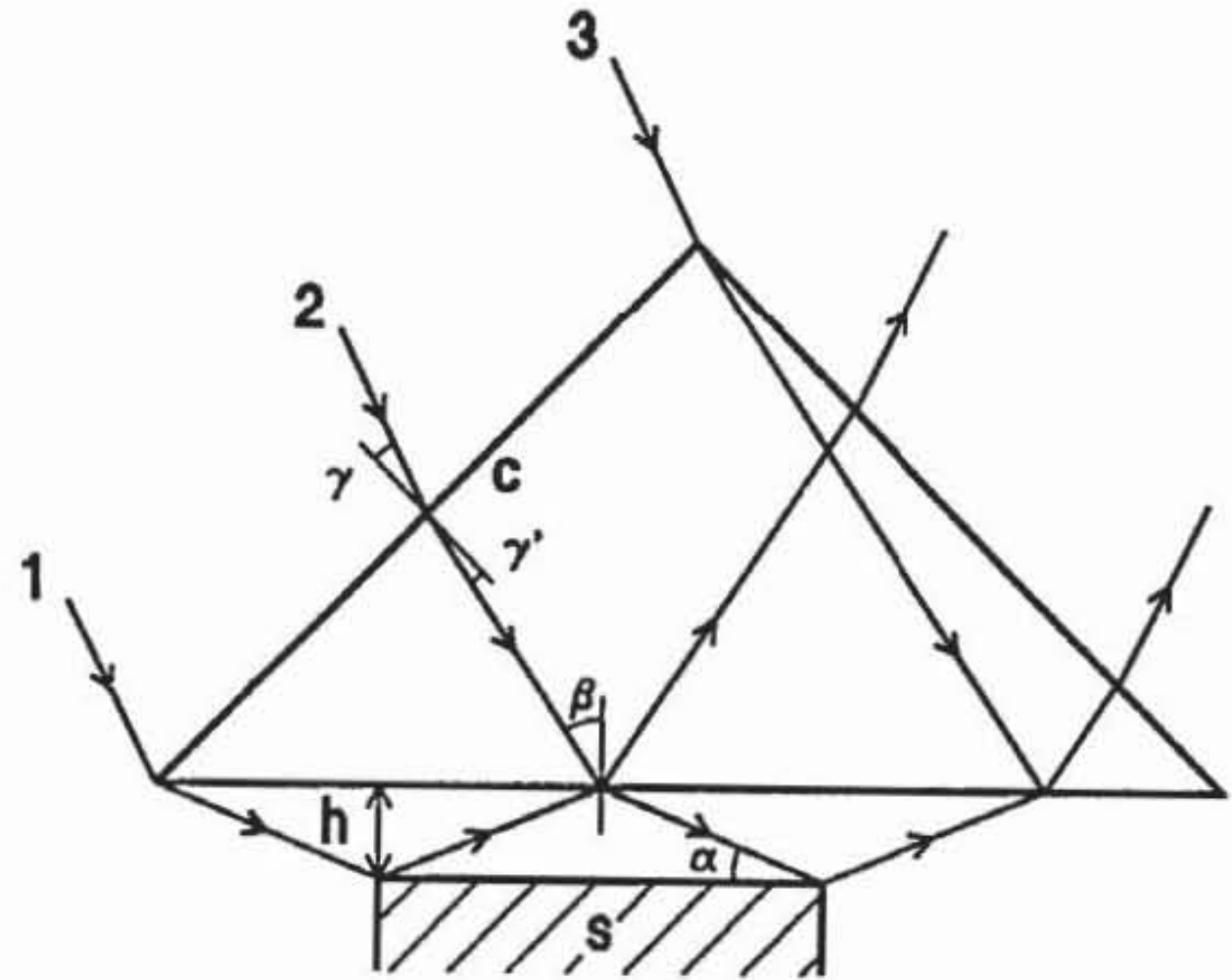

Fig. 3. Avoidance of multiple beam interference and generation of cosinusoidal interference fringes.

Another possibility for obtaining cosinusoidal fringes is to introduce a tilt between the reference and the test surfaces. ${ }^{5}$ The multiple reflections can then be blocked in the focal plane of a lens. A moiré interferometer is used to obtain the interference pattern without the linear carrier due to the tilt.

\section{PHASE CALCULATION AND DECOMPRESSION OF IMAGE DEFORMATION}

To calculate the phase of the wavefront a four-step phase-shift algorithm is used. ${ }^{6,7}$ The intensity distribution of the $i$ 'th interferogram can be expressed as

$I_{i}(x, y)=I_{0}(x, y)\left\{1+m(x, y) \cos \left[\phi(x, y)+\delta_{i}\right]\right\}, \quad i=1 \ldots . .4$,

where $I_{0}(x, y)$ is the average intensity at each point, $m(x, y)$ is the modulation, $\phi(x, y)$ is the phase of the wavefront, and $\delta$ is the introduced phase shift. With a phase shift of $\delta_{i}=-3 \pi / 4$, $-\pi / 4, \pi / 4$, and $3 \pi / 4$, the phase can be calculated from

$\phi(x, y)=\arctan \left[\frac{I_{4}(x, y)-I_{2}(x, y)}{I_{1}(x, y)-I_{3}(x, y)}\right]$

The required phase shift is achieved by changing the thickness of the air gap between the surface under test and the prism hypotenuse. To achieve a phase step of $\pi / 2$ the distance has to be changed by an amount of $\Delta h=\lambda_{\text {eff }} / 8$. To use the whole sensitivity range, we need a displacement of the surface under test of at least $5 \mu \mathrm{m}$. For an accurate phase calculation, the displacement of the surface under test needs to be controlled to lead to a minimum deviation from $\delta_{i}$.

To move the surface under test perpendicular to the reference surface a piezoelectric transducer is integrated into the sample mounting. The variable fringe density of this interferometer requires a calibration of the phase shift. After every sensitivity change, we measure the intensity at a point while shifting the phase in small steps. The intersection points of the intensity profile with the average intensity correspond to phase steps of 0,180 , and $360 \mathrm{deg}$, whereas the extremes between these points correspond to phase steps of 90 and $270 \mathrm{deg}$. The movement of 
the piezoelectric transducer in the sample mounting was calibrated with a Michelson interferometer.

The required phase shift for phase calculation is achieved by moving the surface under test perpendicular to the reference surface. This movement of the sample leads to small lateral shifts between different interferograms. A test beam reflected at $B$ of the surface under test (Fig. 4) crosses the reference surface at $C$. After changing the thickness of the air gap a test beam reflected at $B$ crosses the reference surface at $C^{\prime}$. The lateral shift $v$ from $C$ to $C^{\prime}$ of the crossing point is given by

$v=\Delta h \frac{1}{\tan \alpha}$,

with $\Delta h$ the air gap variation and $\alpha$ the angle of the incident beam. Thus the lateral shift while changing the phase by an amount of $\pi / 2$ is given by

$v=\frac{\lambda_{\mathrm{eff}}}{8} \frac{1}{\tan \alpha}$.

With the local slope of the surface under test $\partial h / \partial x$ the lateral shift $v$ results in a phase difference

$\phi_{\nu}=\frac{\pi}{2 \tan \alpha} \frac{\partial h}{\partial x}$.

With this additional phase difference Eq. (6) can be written as

$\phi^{\prime}=\arctan \left[\frac{\sin \left(\phi+3 \phi_{v}\right)+\sin \left(\phi+\phi_{v}\right)}{\cos (\phi)+\cos \left(\phi+2 \phi_{v}\right)}\right]$.

The residual phase error between the original phase of the wavefront $\phi$ and the calculated phase $\phi^{\prime}$ is shown in Fig. 5 .

In oblique incidence interferometers, the image of the sample is compressed in the direction of oblique incidence. In the prism interferometer, the compression of the sample is reduced by the refraction of the wavefront at the hypotenuse of the prism. The size ratio $r$ between compressed and uncompressed axis is given by

$r=\frac{\cos \gamma}{\cos \gamma^{\prime}} \cos \beta$.

Without the decompression by the refraction of the glass, $r$ is given by

$r=\sin \alpha=\frac{\lambda}{\lambda_{\text {eff }}}$.

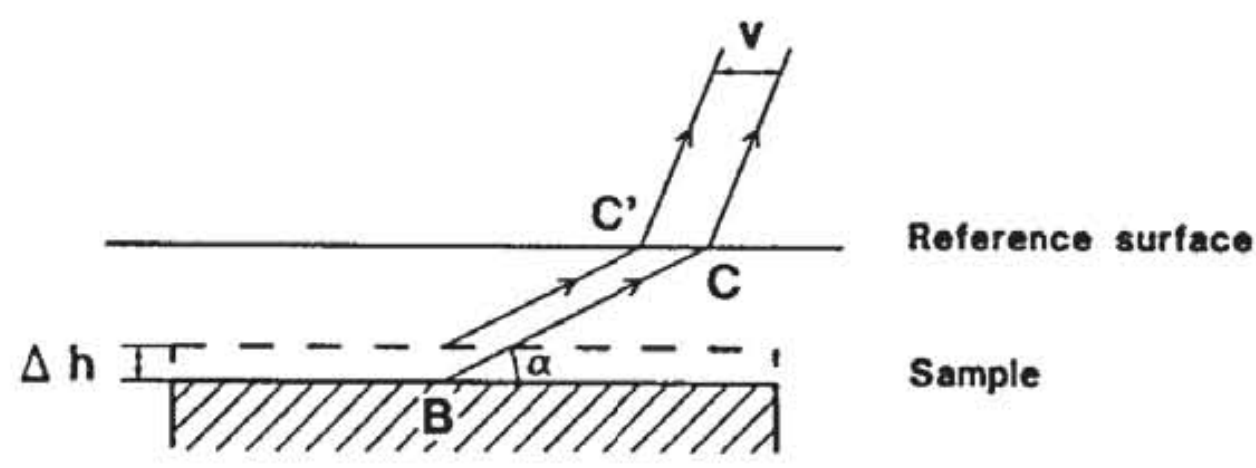

Fig. 4. The lateral shift of the wavefront reflected at the test surface due to the displacement for phase shifting.

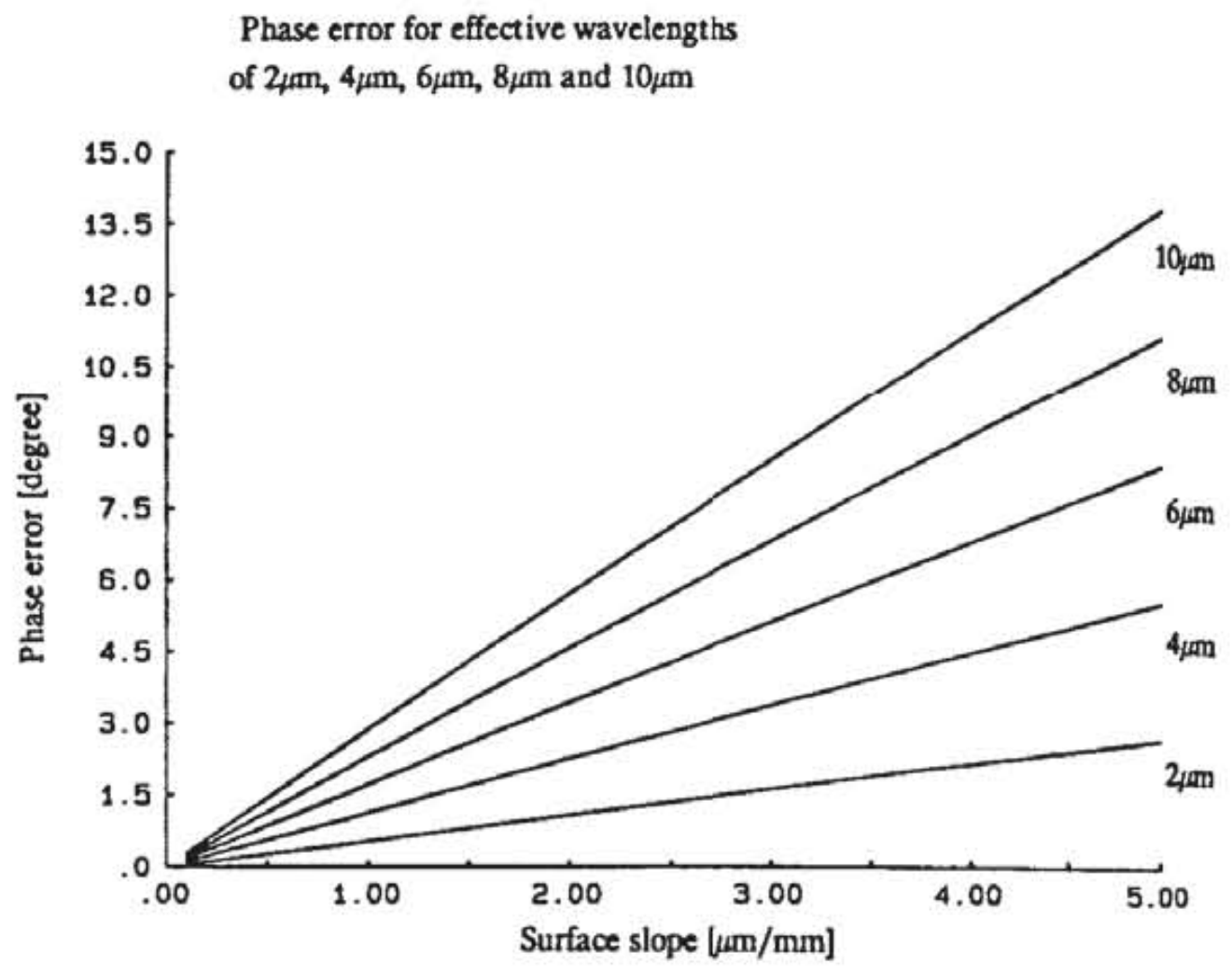

Fig. 5. Phase errors resulting from the lateral shift $v$ as a function of the local surface slope and the effective wavelength as parameter.

Figure 6 shows the size ratio as a function of the effective wavelength in both cases.

After the calibration of the phase shift the effective wavelength is known and this allows the calculation of the remaining compression. During the evaluation of the interference pattern the $x$ axis is spread accordingly and the distortion is decompressed in the presentation of the measurements.

\section{EXPERIMENTAL RESULTS}

In a first example, the topography of a ceramic sample was analyzed. Figures 7 (a) through 7 (c) show resulting interference patterns for different effective wavelengths. The vertical tilt was adjusted for equal number of fringes in the right part of the interferogram. For an effective wavelength of $3.0 \mu \mathrm{m}$ [Fig. 7(a)] the fringe density in the left part of the interference pattern was too high to be evaluated. Other problems occurred from the high local fringe densities due to fine grooves in the ceramic. The slope of the wavefront was reduced by the cure of a longer effective wavelength of $5.2 \mu \mathrm{m}$ [Fig. 7(b)]. The topography was measured with $\lambda_{\text {eff }}=7.1 \mu \mathrm{m}$ [Fig. 7(c)]. A pseudo-threedimensional plot is shown in Fig. 7(d). The reduced sensitivity

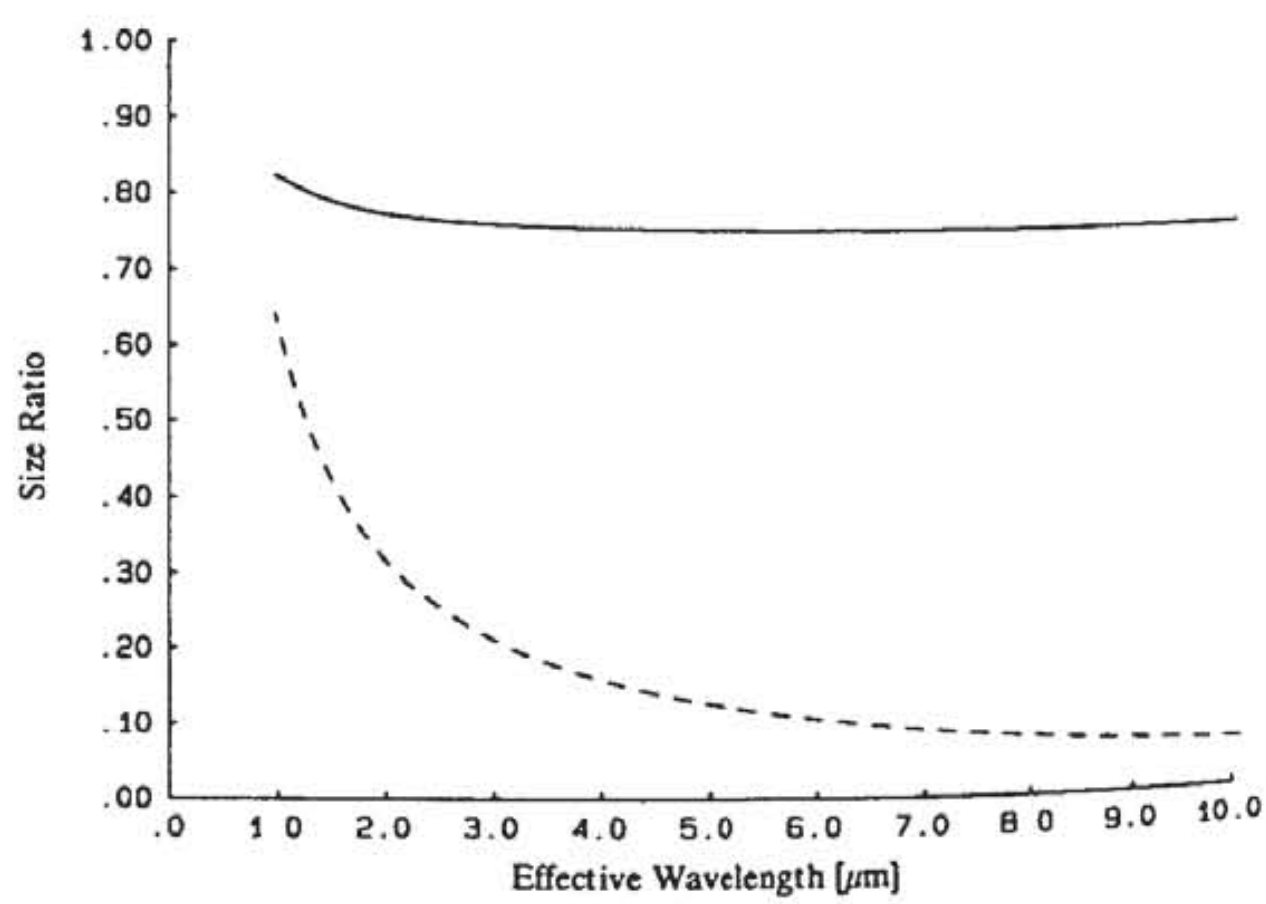

Fig. 6. Image compression in oblique incidence interferometers. The continuous line shows the size ratio in a prism interferometer, whereas the dashed line shows the size ratio by the oblique incidence itself. 


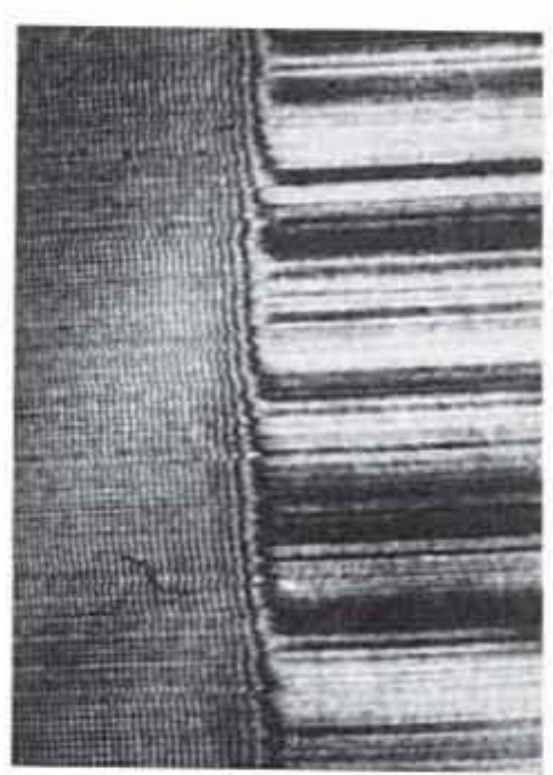

(a)

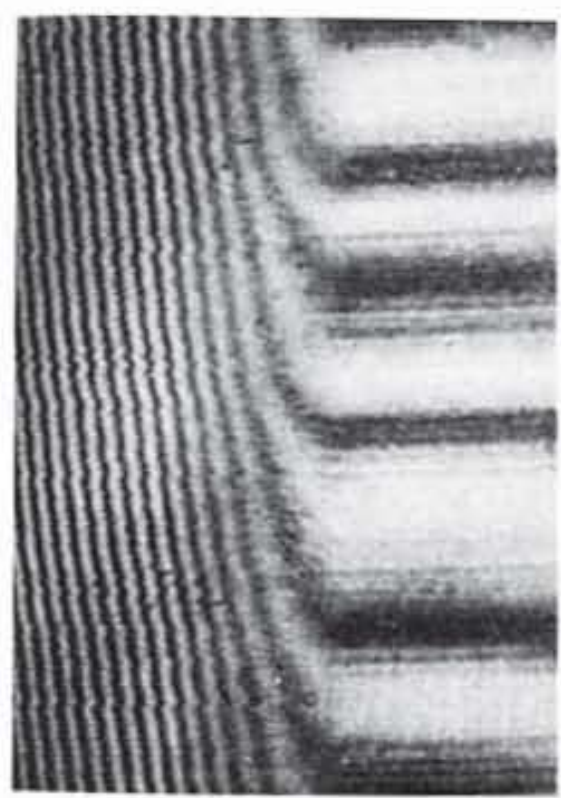

(c)

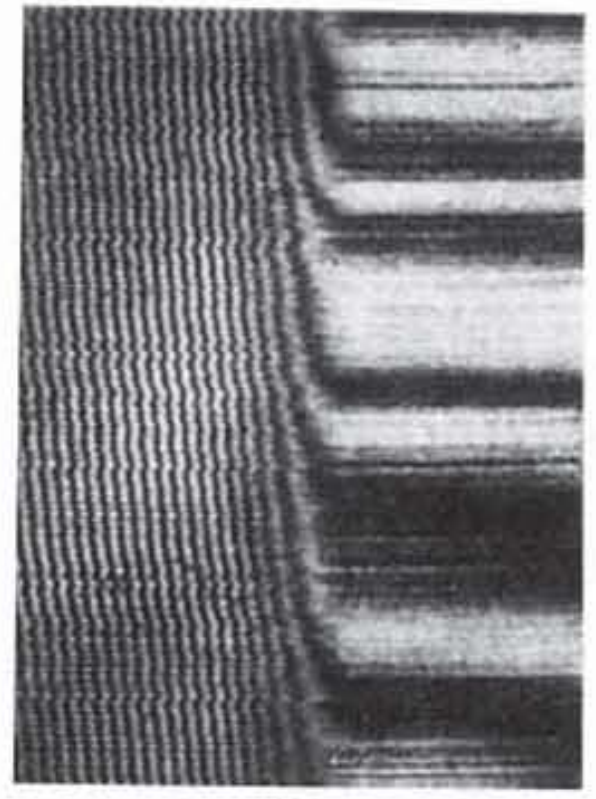

(b)

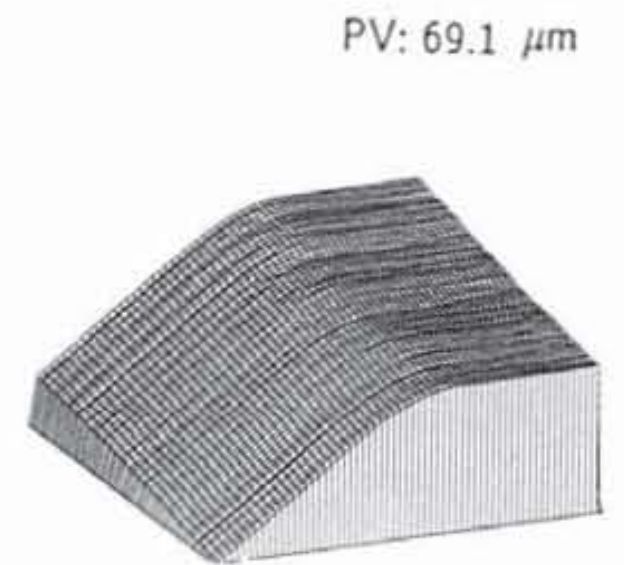

(d)
Fig. 7. Interferogram of a ceramic sample at effective wavelengths of (a) $3.0 \mu \mathrm{m}$, (b) $5.2 \mu \mathrm{m}$, and (c) $7.1 \mu \mathrm{m}$; (d) a pseudo-3-D plot of the sample.

enables the measurement of the height difference of the test surface of about $70 \mu \mathrm{m}$. The size of the measured area was $12 \times 12 \mathrm{~mm}^{2}$. To analyze the surface structure a second measurement of a portion of the surface was made by using an effective wavelength of $5.2 \mu \mathrm{m}$. A pseudo-three-dimensional plot (Fig. 8) shows the parallel abrasion grooves on the surface. The variable fringe separation of this oblique incidence interferometer makes it possible to test the entire sample at low resolution with the ability to focus on portions of the sample in order to analyze fine structures with high resolution.

The second example shows the topography of a steel tappet. With an adjusted effective wavelength of $9.2 \mu \mathrm{m}$ it was possible to resolve the fringes over the whole interference pattern for the automatic phase measurement. A profile and a pseudo-threedimensional plot of the surface are shown in Fig. 9.

\section{CONCLUSION}

The combination of a prism interferometer with a fringe analysis technique allows the measurement of technical surfaces. The phase shifting was done by moving the test surface perpendicular to the hypotenuse of the prism. The phase error resulting from the lateral shift while changing the phase is small compared to the measuring range. With a calibrated shifting device the effective wavelength and the correction factor for image deformation was determined out of the displacement needed for the

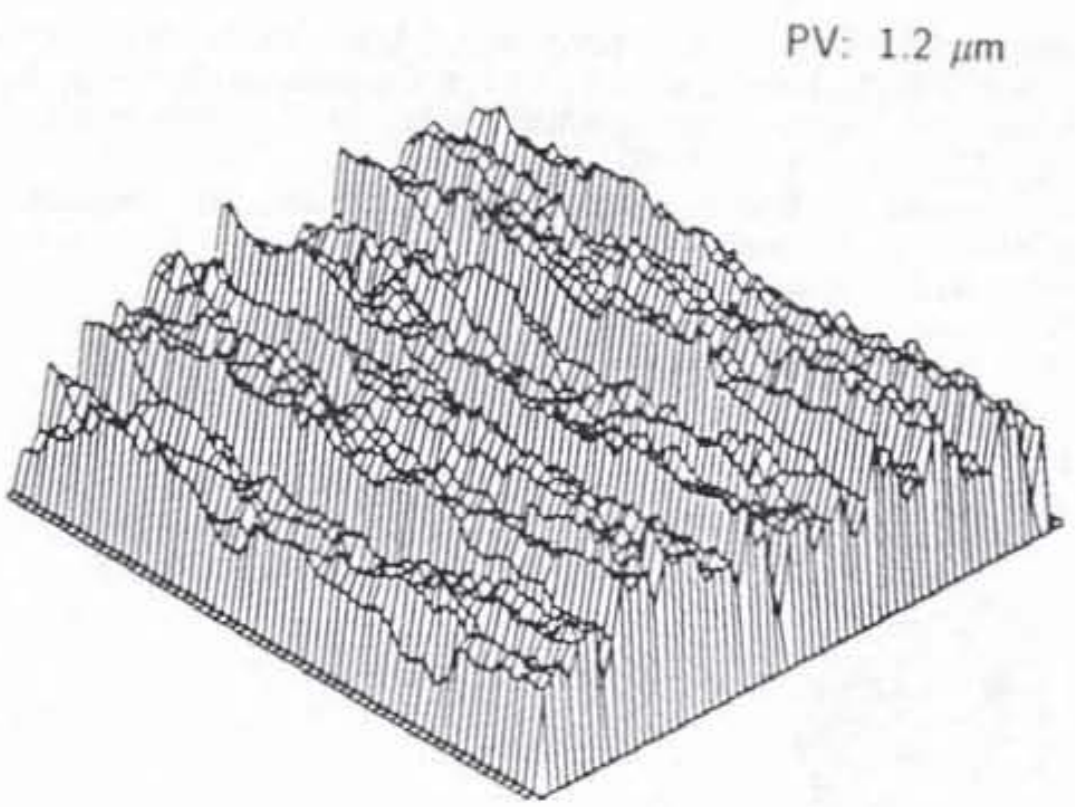

Fig. 8. Pseudo-3-D plot of the surface structure.

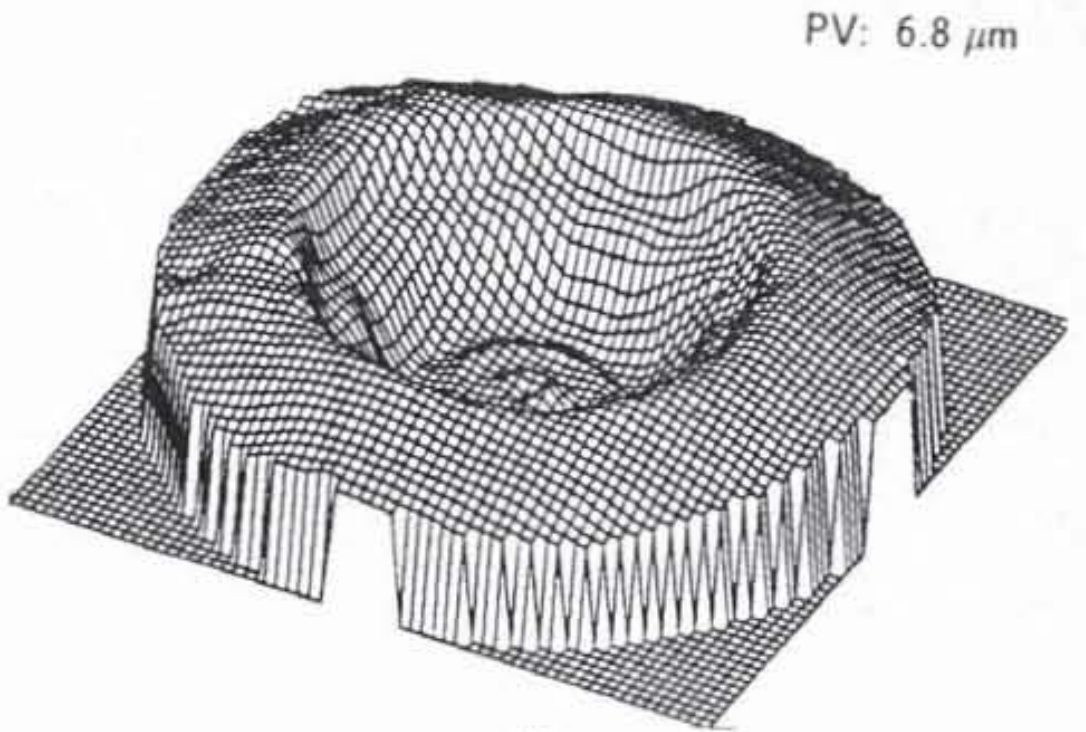

(a)

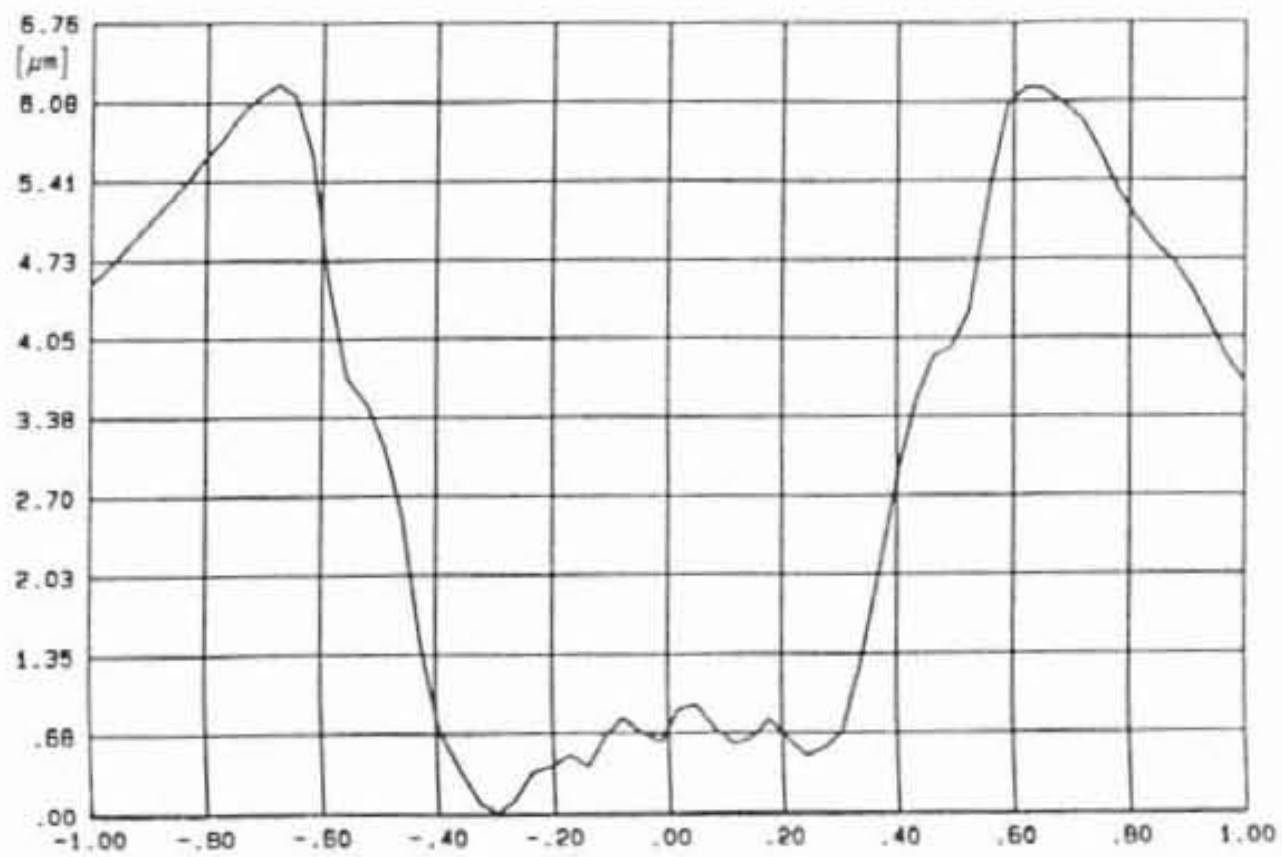

(b)

Fig. 9. Topography of a steel tappet ( $\$ 24 \mathrm{~mm}$ ): (a) pseudo-3-D plot; (b) profile map.

phase shift algorithm. Interference with oblique incidence not only reduces the number of interference fringes but also increases the range of measurable surfaces to rougher surfaces.

\section{REFERENCES}

1. N. Abramson, "The Interferoscope: a new type of interferometer with variable fringe separation," Optik 30, 56-71 (1969).

2. J. D. Briers, "Interferometric flatness testing of nonoptical surfaces," Appl. Opt. 10, 519-524 (1971).

3. K. G. Birch, "Application of the interferoscope to spherical and aspherical surfaces," Optik 36, 399-409 (1972)

4. M. D. A. MacBean. "Oblique incidence interferometry of rough surfaces 
using a novel Dove-prism spectrometer," Appl. Opt, 23, 4024-4028 (1984).

5. J. Schwider, R. Burow, K.-E. Elssner, J. Grzanna, and R. Spolaczky, "Semiconductor wafer and technical flat planeness testing interferometer," Appl. Opt. 25, 1117-1121 (1986).

6. J. Schwider, R. Burow, K.-E. Elssner, J. Grzanna, R. Spolaczky, and K. Merkel, "Digital wave-front measuring interferometry: Some systematic error sources," Appl. Opt, 22, 3421-3432 (1983).

7. K. Creath, "Comparison of phase-measurement algorithms," Proc. SPIE $680,19-28(1986)$.

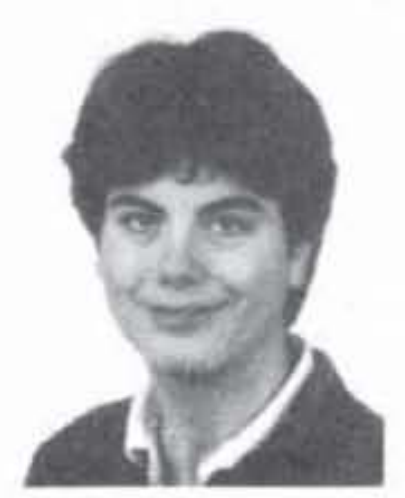

Doris Boebel received her MS degree in mechanical engineering at the University of Stuttgart in 1989. After graduation, she worked in the field of grazing incidence interferometry. At present, she is working in the automotive lighting development group of Bosch.

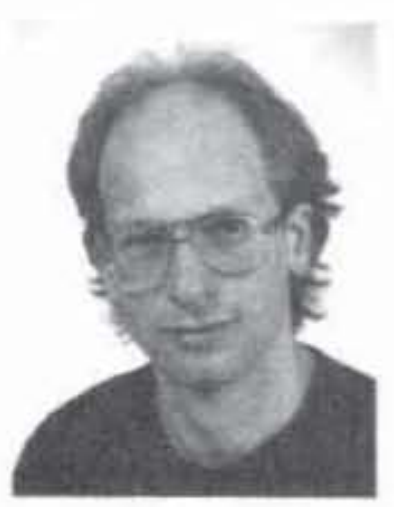

Bernd Packro $\beta$ received his diploma in physics at the University of Essen in 1984. During his diploma thesis, he worked on the selfimaging effect. In 1985 he joined the Institut für Technische Optik as a research worker in the field of interferometric testing of aspheric optics and fringe analysis. Currently, he is working on interferometry with diode lasers.

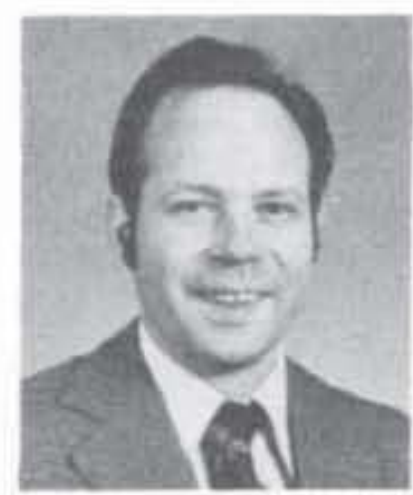

Hans J. Tiziani is director of the Institute of Applied Optics and Professor at the University of Stuttgart. He received his Dipl.-Ing. degrees in mechanical engineering from St. Gallen and in optics from the "Institut d'Op. tique" of Paris and the Ph.D. degree from the Imperial College London and "Habilitation" from the ETH Zürich. He has been a consultant at IBM, San Jose, and head of the Optics Group at the Institute of Applied Physics, ETH Zürich. He is a past president of European Electro-optics and of the Optics Division of the European Physical Society. He is a fellow of SPIE. His main interests are in the fields of image formation, holography and speckle applications, optical system and transfer function analysis and measurement, interferometry, computer generated holography, metrology and inspection, and surface profile measurements. He has written nearly 100 publications on applied optics, optical metrology, and optical surface measurement techniques. 